Arq. Bras. Med. Vet. Zootec., v.53, n.6, p.701-707 2001

\title{
Diferenças entre produções de leite e de gordura de vacas PC e PO da raça Holandesa no Estado de Minas Gerais
}

[Differences on milk and fat yield between purebred and grade Holstein cows in Minas Gerais, Brazil]

\author{
M.C. Durães ${ }^{1}$, J. Valente $^{1}$, A.F. Freitas ${ }^{1}$, N.M. Teixeira ${ }^{1}$, R.B. Barra ${ }^{2}$ \\ ${ }^{1}$ Embrapa Gado de Leite \\ Rua Eugênio do Nascimento, 610 - Bairro Dom Bosco \\ 36038-330 - Juiz de Fora, MG \\ ${ }^{2}$ Associação de Criadores de Gado Holandês de Minas Gerais - Juiz de Fora, MG
}

Recebido para publicação em 11 de setembro de 2000

Recebido para publicação, após modificações, em 17 de setembro de 2001

E-mail: dcm075@cnpgl.embrapa.br

\section{RESUMO}

As produções de leite e de gordura de vacas da raça Holandesa puras de origem (PO) e puras por cruzamento (PC), com gerações controladas, foram analisadas por meio da metodologia de modelos mistos, utilizando máxima verossimilhança restrita e modelo animal. Foram utilizados dados coletados de 1986 a 1996, num total de 49.666 lactações de 26.822 vacas em 380 rebanhos, 9.468 PO e 17.354 PC. Os efeitos fixos foram rebanho/ano/estação, considerando-se três classes de dois meses em cada estação do ano, isto é, seca (abril - maio, junho - julho, agosto - setembro) e águas (outubro - novembro, dezembro janeiro e fevereiro - março) e dois graus de sangue (PO e PC), além dos efeitos linear e quadrático de idade ao parto. O animal foi considerado como efeito aleatório. Para estimar o ganho genético das produções de leite e de gordura foram utilizadas 18.482 primeiras lactações, 8.938 de vacas PO e 9.544 de vacas PC. Foram também formadas cinco classes de produção: até $4.000 \mathrm{~kg}$, de 4.000 a $6.000 \mathrm{~kg}$, de 6.000 a $8.000 \mathrm{~kg}$, de 8.000 a $10.000 \mathrm{~kg}$ e acima de $10.000 \mathrm{~kg}$. As médias gerais estimadas para produção de leite e de gordura até 305 dias, em duas ordenhas diárias, foram, respectivamente, 5.865,54 e 196,85kg. As médias de produção de leite e de gordura para a mesma classe de idade ao parto foram consistentemente maiores para as vacas PO. As estimativas de ganho genético anual para leite e gordura nas classes de produção citadas foram, respectivamente: 10,52 e $0,33 \mathrm{~kg} ; 8,31$ e $0,25 \mathrm{~kg} ; 8,90$ e $0,29 \mathrm{~kg} ; 11,00$ e $0,36 \mathrm{~kg}$; e 9,50 e $0,36 \mathrm{~kg}$. As médias de produção de leite e de gordura para as vacas de primeira cria foram: 6.084,6 e $205,1 \mathrm{~kg}$ e $5.739,5$ e $191,8 \mathrm{~kg}$ para vacas PO e PC, respectivamente. As estimativas de tendências genéticas de 8,7 e 9,6kg por ano para vacas PO e PC, embora pequenas, refletem aumento na capacidade de produção de leite no período.

Palavras-chave: Bovino leiteiro, Holandês, tendência genética

\begin{abstract}
Milk and fat production of purebred and grade Holstein dairy cows in Minas Gerais State, Brazil were analyzed by, mixed models methodology and restricted maximum likelihood in an animal model. The fixed effects were herd-year-season, genetic composition (purebred and grade) and the linear and quadratic effects of age at calving and the random effect was the animal. A total of 49,666 lactations of 26,822 cows from 380 herds, being 9,468 of purebred and 17,354 of grade were used. Two seasons, each with three classes of two months, dry season (April-May, June-July, August-September) and rainy season (October-November, December-January, February-March) were used. In order to estimate genetic gains, 18,482 records of cows in the first calving, being 8,938 of purebred and 9,544 of grade cows were used.
\end{abstract}




\section{Durães et al.}

The data were grouped in five classes: $4,000 \mathrm{~kg}$, from 4,000 to $6,000 \mathrm{~kg}$, from 6,000 to $8,000 \mathrm{~kg}$, from 8,000 to 10,000 and $10,000 \mathrm{~kg}$ of milk production in 305 days. The overall milk and fat average were $5,865.54$ and $196.85 \mathrm{~kg}$, respectively. The milk and fat averages for the same age classe were consistently larger for the purebred cows. The estimated genetic trend for milk and fat in each class of milk production were: 10.52 and $0.33 \mathrm{~kg}, 8.31$ and $0.25 \mathrm{~kg}, 8.90$ and $0.29 \mathrm{~kg}, 11.00$ and $0.36 \mathrm{~kg}, 9.51$ and $0.36 \mathrm{~kg}$ by year, respectively. The average milk and fat production for cows of first calving were: 6,084.6 and $205.1 \mathrm{~kg}, 5,739.5$ and $191.8 \mathrm{~kg}$ for purebred and grade cows. The estimated genetics trends of 8.7 and $9.6 \mathrm{~kg}$, for purebred and graded cows, although small, reflect an increasing in milk production potential from 1986 to 1996.

Keyword: Dairy cattle, Holstein, genetic trend

\section{INTRODUÇ̃̃O}

Os animais da raça Holandesa, não-registrados, são identificados como "grade" pela Associação de Criadores da Raça Holandesa nos EUA. A maioria das vacas "grade" possui um simples ancestral identificado. A utilidade das informações desse grupo de animais em programas de melhoramento segundo Norman \& Powell (1983) é limitada, em virtude de apenas um terço das vacas não registradas em testes oficiais terem o pai identificado.

Em geral as vacas registradas produzem maior quantidade de leite do que as vacas "grade" aos dois anos de idade. Essa superioridade diminui e chega a desaparecer nas lactações seguintes em virtude da maior intensidade de descarte em vacas nesse último grupo (Powell \& Norman, 1986).

No Brasil, a Associação Brasileira de Criadores de Bovinos da Raça Holandesa (ABCBRH) adotou critérios diferentes em relação à sua congênere americana. O gado nacional registrado e importado é considerado como puro de origem (PO) e o gado obtido por cruzamento absorvente é registrado como puro por cruzamento (PC) quando atinge a fração 31/32 de Holandês. A partir dessa fração, as gerações são controladas e os animais são identificados como GC1, GC2 etc. As vacas GC1 são aquelas com pais conhecidos, as GC2 com os pais e os avós, e assim sucessivamente. Por qualificação fenotípica (classificação linear superior a 78 pontos) e produtiva (livro de mérito), vacas Holandesas GC3 em diante podem ser registradas como puras de origem nacional.

O estudo comparativo dos grupos PO e PC é de grande importância para as associações de criadores de gado Holandês. A principal utilidade é a orientação a ser dada aos seus associados quanto ao uso de um ou de ambos os grupos. O objetivo do presente trabalho é comparar as produções de leite e de gordura de vacas Holandesas PO e PC do Estado de Minas Gerais.

\section{MATERIAL E MÉTODOS}

Foram utilizados dados coletados de 1986 a 1996 pelo Serviço de Controle Leiteiro dos núcleos filiados à Associação de Criadores de Gado Holandês de Minas Gerais. Após a edição dos dados, utilizaram-se 49.666 lactações de 26.822 vacas mantidas em 380 rebanhos. As vacas foram agrupadas segundo sua composição racial em puras por cruzamento (PC) e puras de origem (PO), cada grupo constituído, respectivamente, de 17.354 e 9.468 vacas.

As estações da seca (abril-setembro) e das águas (outubro-março) foram subdivididas em períodos de dois meses cada (classe de estação), respectivamente, abril-maio, junho-julho, agosto-setembro e outubronovembro, dezembro-janeiro, fevereiro-março, para minimizar os efeitos das variações climáticas dentro de cada estação, levando-se em conta a extensão do Estado de Minas Gerais. 
As análises estatísticas foram realizadas pela metodologia dos modelos mistos, usando máxima verossimilhança restrita (REML) por meio do sistema MTDFREML (Boldman et al., 1995), adotando-se o seguinte modelo animal:

$\mathbf{Y}=\mathbf{H h}+\mathbf{Z a}+\mathbf{Z p}+\boldsymbol{\varepsilon}$, em que:

Y é o vetor das observações da produção de leite ou gordura até 305 dias, $\mathbf{H}$ é a matriz de incidência dos efeitos fixos rebanho/ano/estação, composição genética (PC, PO), efeitos linear e quadrático da idade da vaca ao parto, $\mathbf{Z}$ a matriz de incidência dos efeitos aleatórios, $\boldsymbol{\varepsilon}$ o vetor de efeitos residuais, $\mathbf{h}$ o vetor de efeitos fixos, a o vetor de efeitos aleatórios do animal (vaca, pai, mãe) e p o vetor de efeitos permanentes inerentes à repetição das observações de cada vaca.

Um subgrupo de dados constituídos de 18.482 registros de produção de vacas da raça Holandesa (PO e PC), que encerraram a primeira lactação até 305 dias e nascidas no mesmo período, foi utilizado para estimar a tendência genética.

Para verificar se houve diferença no ganho genético de acordo com a faixa de produção de leite, os animais foram agrupados por classe de produção: até $4.000 \mathrm{~kg}$, de 4.000 a $6.000 \mathrm{~kg}$, de 6.000 a $8.000 \mathrm{~kg}$, de 8.000 a $10.000 \mathrm{~kg}$ e acima de $10.000 \mathrm{~kg}$.

As estimativas de tendências genéticas de leite e gordura foram obtidas por meio da regressão ponderada dos valores genéticos das vacas sobre o ano de seu nascimento para cada classe.

\section{RESULTADOS E DISCUSSÃO}

As médias de produção de leite para uma mesma classe de idade foram consistentemente maiores para as vacas PO em relação às vacas PC. A amplitude de variação da produção de leite foi menor no grupo PC, 4.540 a $6.299 \mathrm{~kg}$, do que nas PO, 5.006 e $7.111 \mathrm{~kg}$. As médias de produção de leite em 305 dias e duas ordenhas variaram de 5.447 a $5.722 \mathrm{~kg}$ (águas) e de 5.657 a $5.882 \mathrm{~kg}$ (seca) para as vacas PC e de 5.942 a $6.161 \mathrm{~kg}$ (águas) e de 6.206 a $6.303 \mathrm{~kg}$ (seca) para as vacas PO como mostrado na Tab. 1.

As diferenças de produção de leite possivelmente podem ser explicadas pela melhor qualidade genética das vacas PO. A média de produção de leite foi superior para as vacas PO $(6.303,4 \mathrm{~kg})$ que pariram na época seca, classe junho-julho. Para as PC, a maior produção foi na classe abril-maio $(5.882,2 \mathrm{~kg})$. Houve tendência de as vacas que pariram no período seco apresentarem maior produção em relação às que pariram no período das águas. Provavelmente isso ocorreu em virtude de temperaturas mais amenas no início da lactação na época seca, além de melhor trato com rações e forragens conservadas. Em geral, os produtores alimentam suas vacas de acordo com a produção, independentemente do "grau de sangue", pois nessa época fazem a cota junto aos compradores. As vacas mais novas foram menos afetadas pelo estresse das altas temperaturas do verão do que as vacas de mais idade. Essa mesma tendência foi observada por Durães \& Keown (1991), ao trabalharem com dados de rebanhos de nove estados localizados no Meio-Oeste dos Estados Unidos. Esses autores sugeriram maior concentração de partos para as vacas mais velhas na época mais propícia do ano, evitando-se os efeitos prejudiciais de temperaturas mais elevadas, enquanto as mais jovens poderiam parir em qualquer época do ano com a finalidade de prover leite fresco para a população durante todo o ano.

A média de produção de gordura foi maior nas lactações iniciadas no período de seca e maior para as vacas PO (Tab. 2). Em geral, a correlação entre produção de leite e de gordura é alta. Portanto, os valores mais elevados para a produção de gordura eram esperados no grupo PO em virtude de sua maior produção de leite.

Na Tab. 3 são apresentadas as médias, os desvios-padrão e os valores mínimos e máximos das produções de leite e de gordura de vacas PO e PC de primeira cria. As vacas PO produziram aproximadamente $6 \%$ 


\section{Durães et al.}

mais leite do que as PC. A produção de gordura também foi maior para as PO em cerca de 7\%. No entanto, verifica-se que as médias dos valores genéticos (VG por ano) para leite, $76,2 \mathrm{~kg} \times 56,0 \mathrm{~kg}$, e para gordura, $2,1 \mathrm{~kg}$ x $2,0 \mathrm{~kg}$, foram maiores para os animais PC.

As tendências genéticas para produção de leite de acordo com o "grau de sangue" foram 17,4 e 19,2kg por ano para vacas PO e PC, respectivamente. O maior ganho genético para as vacas PC reflete, em parte, o uso de touros com maior capacidade prevista de transmissão somente para leite cujo sêmen é, em geral, de menor preço. No mercado, o preço do sêmen de touros com alta capacidade prevista de transmissão para leite, gordura, proteína e características de tipo usualmente é mais alto. Os criadores de rebanhos PO são mais conservadores e escolhem o sêmen de touros com alta confiabilidade, às vezes sacrificando a capacidade de transmissão prevista para produção de leite em favor de animais de melhor conformação e tipo. Essa mesma tendência foi observada nos EUA, onde os produtores de leite de gado "grade" já estão obtendo maior produção em comparação à de gado registrado, como relatado por Powell \& Norman (1986). Também Durães \& Keown (1991) observaram que as vacas "grade" tiveram produção de leite e gordura semelhantes às vacas PO, com ligeira vantagem para as PC. Em geral, os produtores escolhem sêmen com alto diferencial de produção (PTA mais elevado) para leite e de menor preço para ser indistintamente utilizado nas vacas, exceto para aquelas de maior valor que são avaliadas e classificadas linearmente. Nessas, o sêmen utilizado tem preço mais elevado, pois além da produção, o produtor revela interesse pelas características de tipo e utilizam os acasalamentos corretivos, de acordo com o sistema de classificação linear. Usualmente o criador reserva o sêmen de melhor qualidade genética para as vacas mais valiosas, em geral as PO.

A produção de leite de vacas de primeira cria variou de 1.509 a $15.801 \mathrm{~kg}$. O valor máximo de gordura foi $592 \mathrm{~kg}$. 
Diferenças entre produções de leite e de gordura...

Tabela 1. Média de produção de leite $(\mathrm{kg})$ de vacas da raça Holandesa em Minas Gerais segundo as classes de idade e meses de parição

\begin{tabular}{|c|c|c|c|c|c|c|}
\hline \multirow{3}{*}{$\begin{array}{l}\text { Classe de } \\
\text { idade } \\
\text { (meses) }\end{array}$} & \multicolumn{6}{|l|}{ Época do parto } \\
\hline & \multicolumn{3}{|l|}{ Águas } & \multicolumn{3}{|l|}{ Seca } \\
\hline & Out - Nov & Dez-Jan & Fev - Mar & Abr-Maio & Jun - Jul & Ago-Set \\
\hline \multicolumn{7}{|c|}{ VACAS PC } \\
\hline$<24$ & (36) $5.173,1$ & (39) $5.727,8$ & (71) $5.435,9$ & (78) $5.710,7$ & (67) $5.542,9$ & (71) $4.856,7$ \\
\hline $24-27$ & (227) 5.312,6 & (173) $5.471,7$ & (238) 5.576,3 & (363) 5.997,8 & (420) 5.637,1 & (369) 5.505,9 \\
\hline $27-30$ & (408) $5.154,7$ & (380) 5.349,0 & (363) $5.448,0$ & (440) 5.663,5 & (506) 5.522,3 & (520) 5.330,9 \\
\hline $30-33$ & (350) 5.051,6 & (402) 5.087,8 & (419) 5.290,3 & (374) $5.225,0$ & (353) $5.197,0$ & (369) 5.050,9 \\
\hline $33-36$ & (191) 4.539,5 & (202) $4.737,4$ & (265) 5.289,6 & (350) $5.466,4$ & (311) 5.164,9 & (195) 5.015,3 \\
\hline $36-39$ & (171) $5.437,7$ & (169) $5.014,4$ & 5.297,5 & (339) $5.669,7$ & (364) $5.802,3$ & (320) $5.415,3$ \\
\hline $39-42$ & (274) 5.391,8 & (251) 5.494,6 & (257) 5.671,6 & (328) 5.882,4 & (349) 5.907,1 & (360) 5.715,7 \\
\hline $42-45$ & (267) 5.596,5 & (295) 5.386,9 & (351) $5.663,8$ & (348) 5.782,0 & (346) 5.860,4 & (289) 5.623,7 \\
\hline $45-48$ & (209) 5.465,8 & (201) 5.699,0 & (316) $5.740,8$ & (368) 5.780,7 & (315) 5.779,3 & (224) $5.674,3$ \\
\hline $48-51$ & (158) $5.470,0$ & (134) $5.577,5$ & (226) 5.804,6 & (345) $5.856,4$ & (311) $5.923,8$ & (263) $5.834,4$ \\
\hline $51-54$ & (217) $5.612,1$ & (188) 5.646,8 & (219) $6.133,0$ & (273) 6.047,6 & (236) 6.208,9 & $6.165,1$ \\
\hline $54-57$ & (186) 5.697,8 & (214) $5.751,3$ & (289) $5.835,8$ & (289) 6.035,8 & 6.000,2 & (208) 5.907,9 \\
\hline $57-60$ & (128) 5.728,0 & (156) $5.545,6$ & (257) $6.075,1$ & $6.168,3$ & (249) $5.974,2$ & (178) $5.938,7$ \\
\hline $60-63$ & (113) 5.795,1 & (124) $5.750,3$ & 6.078,0 & 6.201,5 & (226) 5.983,6 & (211) 5.895,5 \\
\hline $63-69$ & (280) 5.783,3 & (263) $5.839,8$ & (347) 5.966,8 & 6.290,3 & 6.299,4 & 6.099,4 \\
\hline $69-75$ & (161) $5.766,7$ & (183) 5.699,0 & $6.134,0$ & (438) 6.203,6 & (357) $6.203,5$ & (265) $5.924,3$ \\
\hline $75-81$ & (213) 5.604,2 & (188) 5.732,0 & 6.090,8 (229) & (246) $6.256,7$ & (218) $6.117,8$ & (218) $5.997,8$ \\
\hline $81-87$ & (106) 5.800,2 & (92) $6.077,2$ & (216) $6.151,3$ & (287) $5.938,7$ & 6.091,9 & 6.083 .9 \\
\hline $87-93$ & (130) 5.678,0 & (141) $5.615,5$ & (127) $5.535,3$ & (162) $5.976,5$ & $6.286,6$ & (122) $5.817,5$ \\
\hline $93-99$ & (71) $5.868,8$ & (76) $5.568,3$ & (122) $5.869,2$ & (186) $6.191,5$ & (164) 5.943,9 & (106) $5.770,9$ \\
\hline$>99$ & (232) $5.471,9$ & (267) $5.294,2$ & (369) $5.443,0$ & (431) $5.550,2$ & (368) 5.800,3 & (295) 5.673,5 \\
\hline Média & $5.446,9 \pm 28$ & $5.466,5 \pm 26$ & $5.721,7 \pm 27$ & $5.882,2 \pm 25$ & $5.836,8 \pm 28$ & $5.656,8 \pm 32$ \\
\hline \multicolumn{7}{|c|}{ VACAS PO } \\
\hline$<24$ & (45) $5.404,1$ & (41) $5.703,3$ & (53) $5.824,2$ & (52) $5.947,5$ & (65) 5.794,7 & (44) $5.501,1$ \\
\hline $24-27$ & (234) $5.847,7$ & (172) $5.734,9$ & (231) $5.998,1$ & (287) 6.164 .6 & 6.099,0 (378) & (317) $5.955,2$ \\
\hline $27-30$ & (281) 5.728,2 & (305) 5.750,9 & (285) $5.820,2$ & (352) $6.088,1$ & 6.030,3 & (343) $5.831,2$ \\
\hline $30-33$ & (171) $5.356,9$ & (188) $5.464,2$ & (261) $5.675,5$ & (285) $5.767,8$ & (218) $5.666,1$ & (181) 5.610,0 \\
\hline $33-36$ & (84) $5.005,8$ & (83) $5.701,6$ & (154) $5.588,2$ & (179) $5.418,7$ & (142) $5.840,7$ & (116) 5566,1 \\
\hline $36-39$ & (103) 6.096,2 & (105) $6.029,7$ & (108) $5.856,9$ & 5.986,5 & (213) $6.146,1$ & (182) $6.107,4$ \\
\hline $39-42$ & (184) 6.040,5 & (148) $6.051,0$ & (173) $6.148,8$ & (206) 6.077,6 & (221) $6.435,7$ & $6.379,5$ \\
\hline $42-45$ & (133) 6.010,2 & (147) $6.088,1$ & (219) $6.340,8$ & (237) $6.169,1$ & (172) $6.220,1$ & (174) $6.429,0$ \\
\hline $45-48$ & (97) $5.898,5$ & (88) $6.102,5$ & (166) $6.547,5$ & (184) 6.668,6 & 6.292,5 & (111) $6.155,1$ \\
\hline $48-51$ & (81) $6.221,3$ & (88) $5.938,7$ & (110) 6.282,2 & $6.780,0$ & (150) 6.413,0 & (125) $6.573,8$ \\
\hline $51-54$ & (99) $6.282,7$ & (106) $6.158,3$ & (114) $6.502,6$ & (130) $6.432,5$ & (135) 6.605,4 & (141) 6.632,6 \\
\hline $54-57$ & 6.439,3 & (98) $6.246,3$ & (153) $6.282,4$ & (185) $6.291,8$ & (124) $6.231,2$ & (119) $6.707,4$ \\
\hline $57-60$ & (68) $6.156,9$ & (64) $6.362,4$ & (120) 6.792,4 & (172) 6.395,8 & (142) 6.652,0 & (118) 6.704,7 \\
\hline $60-63$ & (67) $6.347,5$ & (59) $6.386,2$ & (95) $6.304,1$ & (122) $6.490,2$ & 7.110,8 & (99) $6.217,8$ \\
\hline $63-69$ & (133) $6.210,1$ & (128) $6.127,3$ & (158) $6.433,4$ & $6.417,7$ & 6.947,5 & $6.604,9$ \\
\hline $69-75$ & 6.374,1 & (96) $5.932,8$ & (137) $6.477,0$ & $6.796,7$ & (179) 6.927,2 & (136) $6.758,5$ \\
\hline $75-81$ & (106) $6.434,4$ & (92) $6.112,0$ & (121) $6.211,1$ & (140) $6.684,8$ & (121) $6.605,1$ & $6.250,6$ \\
\hline $81-87$ & (67) $6.239,4$ & (64) $6.227,6$ & (94) $6.371,6$ & 6.819,3 & (112) $6.746,2$ & (75) $6.843,2$ \\
\hline $87-93$ & (53) $6.207,4$ & (70) $5.994,0$ & (71) $6.466,8$ & (89) $6.479,0$ & (70) $6.225,7$ & (61) $6.246,9$ \\
\hline $93-99$ & (48) $6.055,2$ & (34) $5.574,9$ & (58) $6.300,0$ & (83) $6.286,0$ & (72) $6.481,3$ & (44) $6.676,7$ \\
\hline$>99$ & (127) $5.644,3$ & (136) $5.930,6$ & (170) $6.145,6$ & (188) $5.990,4$ & (194) $5.972,8$ & (172) $5.795,9$ \\
\hline Média & $5.957,4 \pm 33$ & $5.941,7 \pm 22$ & $6.161,2 \pm 27$ & $6.251,0 \pm 32$ & $6.303,4 \pm 34$ & $6.206,0 \pm 36$ \\
\hline
\end{tabular}

Número de observações entre parênteses 


\section{Durães et al.}

Tabela 2. Média de produção de gordura $(\mathrm{kg})$ de vacas da raça Holandesa no Estado de Minas Gerais, segundo as classes de idade e meses de parição

\begin{tabular}{|c|c|c|c|c|c|c|}
\hline \multirow{3}{*}{$\begin{array}{l}\text { Classes de idade } \\
\text { (meses) }\end{array}$} & \multicolumn{6}{|c|}{ Época do parto } \\
\hline & \multicolumn{3}{|c|}{ Águas } & \multicolumn{3}{|c|}{ Seca } \\
\hline & Out. - Nov. & Dez. - Jan. & Fev. - Mar. & Abr.- Maio & Jun. - Jul. & Ago. - Set. \\
\hline \multicolumn{7}{|l|}{ Vacas PC } \\
\hline$<24$ & (35) 182,1 & (39) 191,1 & (71) 181,2 & (78) 192,7 & (67) 184,0 & (71) 166,3 \\
\hline $24-27$ & (227) 178,6 & (173) 181,6 & (237) 187,1 & (363) 195,8 & (420) 187,7 & (369) 185,0 \\
\hline $27-30$ & (407) 173,6 & (380) 180,4 & (363) 182,5 & (440) 187,0 & (506) 185,1 & (520) 179,5 \\
\hline $30-33$ & (350) 170,3 & (401) 170,9 & (419) 177,4 & (374) 176,5 & (353) 174,0 & (369) 169,5 \\
\hline $33-36$ & (191) 155,0 & (202) $160, .3$ & (265) 177,0 & (350) 180,9 & (311) 173,5 & (195) 170,5 \\
\hline $36-39$ & (171) 182,6 & (169) 168,5 & 178,5 & (339) 191,5 & (364) 190,6 & (320) 182,0 \\
\hline $39-42$ & (274) 180,3 & (250) 187.3 & (257) 191,1 & (328) 195,7 & (349) 197,7 & (360) 191,4 \\
\hline $42-45$ & (267) 187,0 & (295) 183,1 & (351) 190,7 & (348) 193,9 & (346) 196,8 & (289) 188,2 \\
\hline $45-48$ & (209) 182,9 & (201) 192,0 & (316) 193,1 & (368) 192,7 & (315) 195,0 & (224) 190,7 \\
\hline $48-51$ & (158) 182,4 & (134) 181,9 & (226) 193,2 & (345) 195,5 & (311) 197,0 & (263) 193,9 \\
\hline $51-54$ & (217) 187,8 & (188) 188,7 & (219) 203,3 & (273) 201,5 & (236) 207,5 & (230) 205,1 \\
\hline $54-57$ & (186) 189,1 & (214) 191,5 & (289) 195,1 & (288) 201,7 & (196) 197,8 & (208) 198,6 \\
\hline $57-60$ & (128) 192,3 & (156) 188,6 & (257) 204,2 & (330) 205,8 & (249) 200,6 & (178) 199,3 \\
\hline $60-63$ & (113) 195,2 & (124) 194,4 & (166) 202,6 & (279) 206,0 & (226) 200,2 & (211) 200,6 \\
\hline $63-69$ & (280) 192,1 & (263) 197,7 & (347) 200,4 & (392) 208,4 & (344) 209,2 & (273) 204,3 \\
\hline $69-75$ & (161) 192,7 & (183) 191,0 & (290) 204,3 & (438) 207,6 & (357) 205,3 & (265) 199,5 \\
\hline $75-81$ & (213) 188,4 & (188) 194,5 & (229) 206,9 & (246) 209,2 & (218) 204,3 & (218) 200,3 \\
\hline $81-87$ & (106) 194,5 & (92) 204,0 & (216) 207,8 & (287) 200,2 & (247) 205,3 & (170) 205,4 \\
\hline $87-93$ & (130) 188,5 & (141) 185,2 & (127) 185,8 & (162) 200,3 & (140) 208,4 & (122) 194,2 \\
\hline $93-99$ & (71) 196,7 & (76) 189,8 & (122) 199,4 & (186) 204,2 & (164) 199,1 & (106) 193,2 \\
\hline$>99$ & (232) 186,0 & (267) 180,2 & (369) 181,1 & (431) 186,6 & (368) 195,0 & (295) 191,3 \\
\hline Média & $182,8 \pm 0,8$ & $184,1 \pm 0,8$ & $191,9 \pm 0,9$ & $196,2 \pm 0,8$ & $194,9 \pm 0,09$ & $190,0 \pm 1,0$ \\
\hline \multicolumn{7}{|l|}{ Vacas PO } \\
\hline$<24$ & (45) 189,5 & (41) 195,2 & (53) 197,1 & (52) 194,4 & (65) 198,0 & (44) 190,5 \\
\hline $24-27$ & (234) 198,9 & (172) 195,5 & (231) 201,2 & (287) 203,6 & (378) 202,4 & (314) 199,8 \\
\hline $27-30$ & (281) 196,2 & (305) 193,2 & (285) 195,8 & (352) 205,7 & (360) 203,7 & (340) 197,5 \\
\hline $30-33$ & (170) 183,8 & (188) 186,5 & (261) 193,6 & (285) 195,2 & (218) 192,5 & (181) 190,8 \\
\hline $33-36$ & (84) 169,8 & (83) 194,9 & (154) 190,7 & (179) 181,2 & (142) 196,3 & (116) 189,8 \\
\hline $36-39$ & (103) 2085 & (105) 200,5 & (108) 197,6 & (200) 201,5 & (213) 205,5 & (182) 202,5 \\
\hline $39-42$ & (184) 201,1 & (148) 206,4 & (173) 209,5 & (206) 206,2 & (221) 214,5 & (208) 211,6 \\
\hline $42-45$ & (132) 202,2 & (147) 204,4 & (219) 214,8 & (237) 208,4 & (172) 208,0 & (174) 214,4 \\
\hline $45-48$ & (97) 195,5 & (88) 209,5 & (166) 221,8 & (184) 227,0 & (153) 211,0 & (111) 207,9 \\
\hline $48-51$ & (81) 201,5 & (88) 200,6 & (110) 213,9 & (163) 227,1 & (150) 213,6 & (125) 219,7 \\
\hline $51-54$ & (99) 213,2 & (106) 205,2 & (114) 219,2 & (130) 213,2 & (135) 221,8 & (141) 220,3 \\
\hline $54-57$ & (97) 215,2 & 211.5 & (153) 211,9 & (185) 213,6 & (124) 208,3 & (119) 221,0 \\
\hline $57-60$ & (68) 210,5 & (64) 214,5 & (120) 224,9 & (171) 213,2 & (142) 221,7 & (117) 222,7 \\
\hline $60-63$ & (67) 207,9 & (59) 217,4 & (95) 213,7 & (122) 218,2 & (100) 232,3 & (99) 207,1 \\
\hline $63-69$ & (132) 212,4 & (128) 208,3 & (158) 215,8 & (213) 218,4 & (178) 228,2 & (159) 219,1 \\
\hline $69-75$ & (109) 218,7 & (96) 202,0 & (137) 216,2 & (206) 2305 & (178) 230,9 & (136) 225,7 \\
\hline $75-81$ & (106) 215,9 & (92) 205,9 & (121) 207,6 & (140) 223,4 & (121) 218,6 & (106) 211,4 \\
\hline $81-87$ & (67) 210,1 & (64) 210,1 & (94) 215,2 & (108) 229,5 & (112) 222,9 & (74) 232,2 \\
\hline $87-93$ & (53) 208,7 & (69) 203,4 & (71) 216,7 & (89) 218,8 & (70) 208,9 & (61) 210,6 \\
\hline $93-99$ & (47) 203,7 & (34) 195,3 & (58) 209,0 & (83) 215,8 & (72) 219,4 & (44) 220,4 \\
\hline$>99$ & (127) 193,1 & (136) 203,7 & (170) 209,0 & (188) 203,4 & (194) 201,6 & (172) 195,6 \\
\hline Média & $201,6 \pm 1,0$ & $201,4 \pm 0,7$ & $207,9 \pm 0,8$ & $210,6 \pm 1,1$ & $210,7 \pm 1,0$ & $208,0 \pm 1,1$ \\
\hline
\end{tabular}

Na Tab. 4 estão mostrados as médias de produção de leite e gordura na primeira lactação e os respectivos valores genéticos de acordo com a classe de produção. Os valores genéticos para leite aumentaram à medida que a média de produção de leite de cada classe aumentou, chegando ao valor máximo de $116 \pm 162,5 \mathrm{~kg}$. Independentemente da classe de produção, os valores genéticos estimados, tanto para a produção de leite quanto para a produção de gordura, apresentaram valores mínimos negativos, com maior amplitude de variação para a segunda classe de produção (-676 e 987). Os dados revelaram que 
Diferenças entre produções de leite e de gordura...

$75,2 \%$ da produção de leite variou de 4.000 a $8.000 \mathrm{~kg}$ e apenas $13 \%$ das vacas produziram abaixo de $4.000 \mathrm{~kg}$ e $11,8 \%$ acima de $8.000 \mathrm{~kg}$.

As tendências genéticas para a produção de leite e gordura foram: 10,52 e 0,33; 8,61 e 0,25kg; 8,90 e $0,29 \mathrm{~kg} ; 11,00$ e $0,36 \mathrm{~kg} ; 9,50$ e $0,36 \mathrm{~kg}$ por ano, respectivamente, para as cinco classes em ordem crescente de produção.

Tabela 3. Médias, desvios-padrão e valores mínimo e máximo das produções de leite e de gordura e dos valores genéticos de vacas da raça Holandesa, PO e PC de primeira cria

\begin{tabular}{lcccc}
\hline Característica & Médias & Desvios-padrão & Mínimo & Máximo \\
\hline Produção & & & & \\
Leite (PO) & $6.084,6$ & $1.748,0$ & 1.509 & 15.801 \\
Leite (PC) & $5.739,5$ & $1.631,4$ & 1.556 & 15.234 \\
Gordura (PO) & 205,1 & 56,1 & 0 & 592 \\
Gordura (PC) & 191,8 & 48,8 & 27 & 474 \\
Valor genético & & & & 987 \\
Leite (PO) & 56,0 & 151,3 & -676 & 890 \\
Leite (PC) & 76,2 & 146,5 & -569 & 32 \\
Gordura (PO) & 2,0 & 4,7 & -18 & 29 \\
Gordura (PC) & 2,1 & 3,9 & -19 & \\
\hline
\end{tabular}

8.938 observações para PO e 9.544 para PC

Tabela 4. Número de observações, médias, valores mínimo e máximo e valores genéticos de leite e de gordura de acordo com a classe de produção na primeira lactação de vacas da raça Holandesa

\begin{tabular}{|c|c|c|c|c|c|}
\hline \multirow{2}{*}{ Característica } & \multicolumn{5}{|c|}{ Classe de produção } \\
\hline & 4000 & $4000-6000$ & $6000-8000$ & $8000-10000$ & 10000 \\
\hline Número de observações & 2.409 & 7.660 & 6.304 & 1.834 & 275 \\
\hline \multicolumn{6}{|l|}{ Produção de leite } \\
\hline Média & 3.392 & 5.059 & 6.870 & 8.681 & 10.910 \\
\hline Mínimo & 1.509 & 4.000 & 6.000 & 8.000 & 10.001 \\
\hline Máximo & 3.999 & 5.999 & 7.999 & 9.998 & 15.801 \\
\hline \multicolumn{6}{|l|}{ Produção de gordura } \\
\hline Média & 118 & 174 & 228 & 280 & 347 \\
\hline Mínimo & 22 & 27 & 85 & 100 & 129 \\
\hline Máximo & 317 & 317 & 382 & 521 & 592 \\
\hline \multicolumn{6}{|l|}{ Valor genético - leite } \\
\hline Média & 40 & 51 & 84 & 94 & 116 \\
\hline Mínimo & -523 & -676 & -569 & -635 & -427 \\
\hline Máximo & 748 & 987 & 890 & 821 & 795 \\
\hline \multicolumn{6}{|l|}{ Valor genético - gordura } \\
\hline Média & 1,1 & 1,5 & 2,6 & 3,0 & 3,7 \\
\hline Mínimo & -16 & -18 & -19 & -15 & -14 \\
\hline Máximo & 20 & 32 & 29 & 28 & 21 \\
\hline
\end{tabular}

Tendência genética de 9,3 a 10,4kg estimada por Sigurdsson \& Arnason (1995) foram similares, e de $15,5 \mathrm{~kg}$ por Sasaki et al. (1993), um pouco acima dos valores deste trabalho. Teixeira et al. (1994), ao

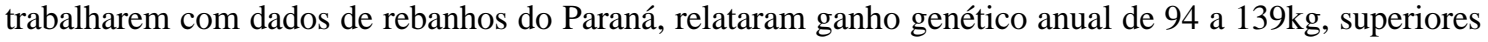
aos obtidos neste estudo. Todavia, esses autores julgaram muito altas essas estimativas em relação aos trabalhos previamente publicados por outros pesquisadores. 


\section{CONCLUSÕES}

As tendências genéticas, apesar de pequenas, refletem aumento da capacidade de produção de leite/vaca durante o período avaliado. Independentemente do nível de produção de cada rebanho, parece haver escolha de sêmen de touros provados visando à melhoria da qualidade dos rebanhos, o que refletiu nos ganhos genéticos positivos apresentados. Possivelmente os criadores de gado PC têm utilizado sêmen de touros provados com maior valor genético para produção de leite e de gordura. Parece que as PO são melhor manejadas e alimentadas que as PC, explicação plausível para sua maior produção de leite e gordura. Mantendo-se essa tendência é possível, a longo prazo, que as vacas PC venham a produzir mais leite e gordura que as PO, a exemplo do que vem ocorrendo nos EUA.

\section{REFERÊNCIAS BIBLIOGRÁFICAS}

BOLDMAN, K.G., KRIESE, L.A., VAN VLECK, L.D. et al. A manual for use of MTDFREML: a set of programs to obtain estimates of variances and covariances. Lincoln: Department of Agriculture/Agricultural Research Service, 1995. 120p.

DURÃES, M.C., KEOWN, J.F. Age-month factors. Mature equivalent factors for three yield traits for non-registered and registered cattle. Rev. Bras. Genet., v.14, p.713-728, 1991.

NORMAN, H.D., POWELL, R.L. Use of grade animals in United States Department of Agriculture programs. $J$. Dairy Sci., v. 66, p. 1567-1578, 1983.

POWELL, R.L., NORMAN, H.D. Genetic and environmental differences between registered and grade Holstein cows. J. Dairy Sci., v. 69, p. 2897- 2907, 1986.

SASAKI, O., TOGASHI, K., YOKOUCHI, K. et al. Analysis of annual genetic gain in milk yield in a dairy herd. Res. Bull. Hokkaido Agric. Exp. Sta., v.58, p.21-30, 1993.

SIGURDSSON, A., ARNASON, R. Predicting genetic trend by uni- and multrait models. Acta Agr. Scand., v.45, p.1-10, 1995.

TEIXEIRA, N. M., FREITAS, A.F., RIBAS, N.P. et al. Tendências genéticas em rebanhos da raça Holandesa no Estado do Paraná. I. Produção de leite. Rev. Soc. Bras. Zootec., v.23, p.983-991, 1994. 